\title{
POSITIVE DEFINITE FUNCTIONALS IN BANACH SPACES
}

\author{
G. G. HAMEDANI \\ (Received 23 August 1976) \\ Communicated by J. Gani
}

\begin{abstract}
We establish a version of Bochner Theorem due to S. Boylan for Banach spaces with a basis.
\end{abstract}

\section{Introduction}

The classical version of Bochner's theorem asserts that a function $\varphi(x)$, $x \in R^{n}$ is the c.f. (characteristic functional or Fourier transform) of some probability measure on the Borel subsets of $R^{n}$ if and only if $\varphi$ is positive definite, $\varphi(0)=1$, and $\varphi$ is continuous at $x=0$. In $l_{2}$, and hence in any real separable Hilbert space, it is possible to introduce a topology $\tau$ (Gross (1963), Sazanov (1958)) such that a function $\varphi$ on $l_{2}$ is the c.f. of some probability measure on the Borel subsets of $l_{2}$ if and only if $\varphi$ is positive definite, $\varphi(0)=1$, and $\varphi$ is continuous at zero (and hence everywhere on $l_{2}$ ) in the $\tau$-topology. Boylan (1975) proved the following version of Bochner's Theorem.

THEOREM B. Assume $\varphi(x)$ is a bounded continuous functional on $l_{2}$ such that $\varphi(0)=1$. Then $\varphi(x)$ is a c.f. of a positive measure on $l_{2}$, if and only if

(a) $T_{t} \varphi(y)=E_{z}^{t \rho}\{\varphi(z) \exp \{i(z, y)\}\} \vee \operatorname{det}(1+t \rho) \geqq 0$ for all $y \in l_{2}$, for all $t \geqq t_{0}$, some $t_{0}>0$.

(b) Setting $\varphi(\gamma x)=\varphi_{\gamma}(x)$, we have

$$
\lim _{y \rightarrow 0} \lim _{t \rightarrow \infty} \int_{l_{2}} T_{t} \varphi_{y}(y) \nu_{1}(d y)=\varphi(0)
$$

where

(i) $\nu_{1}$ is a Gauss measure on $l_{2}$ with variance parameter 1 ,

(ii) $\rho$ is a linear, symmetric, positive, compact and trace class operator on $l_{2}$, 
(iii) $E_{z}^{i p}$ denotes expectation with respect to a Gaussian process $\left\{z: z \in l_{2}\right\}$ with mean zero and covariance $\rho$.

In proving the above theorem, Boylan characterizes positive definite functionals on $l_{2}$ by showing that positive definiteness is equivalent to condition (a). The interesting aspect is that the conditions (a) and (b) use essentially the same entities. The analogue of (a) has been obtained by Boylan (1975) for real separable Banach spaces. However Boylan leaves as an open problem the extension of his version of Bochner's theorem to general spaces. We do this here for Banach spaces with a Schauder basis. We note that the only characterization of positive definiteness we use is through the Hilbert space case, i.e., Theorem B.

\section{Terminology and preliminaries}

Throughout this paper $B$ will denote a real $B$ anach space with Schauder basis $\left\{b_{n}\right\}$ such that without loss of generality $\left\|b_{n}\right\|_{B}=1$ (Day (1962), p. 68). We will write the expansion of $x$ as $\sum_{n=1}^{x} \beta_{n}(x) b_{n}$ and this emphasizes that the coefficients generate coordinate functionals on $B$. It is clear that these coordinate functionals are linear and it is well known that they are continuous as well (Day (1962), p. 68). Further it is possible to assume without loss of generality (Day (1962), Theorem 1, p. 67) that

$$
\|x\|_{B}=\sup _{k}\left\|\sum_{n=1}^{k} \beta_{n}(x) b_{n}\right\|_{B}
$$

We say that $\lambda \in l_{x}^{+}$is adequate for $B$ if

(i) for all $x \in B, \sum_{i=1}^{x} \lambda_{i} \beta_{i}^{2}(x)$ is finite;

(ii) for all $x$ there exists a nonnegative constant $C_{\lambda}$ such that

$$
\sum_{i=1}^{\infty} \lambda_{i} \beta_{i}^{2}(x) \leqq C_{\lambda}\|x\|_{B}^{2}
$$

Here $l_{x}$ denotes the space of bounded sequences. We note at the outset that if $B$ is a Hilbert space then $\lambda=(1,1,1, \cdots)$ is adequate and then $C_{\lambda}=1$.

2.2. Remarks. (a) $\lambda \in l_{1}^{+}$is adequate for $B$, since $\sup _{n}\left|\beta_{n}(x)\right| \leqq k\|x\|_{B}$ Day (1962), p. 68) and $C_{\lambda}=\sum_{i=1}^{\infty} \lambda_{i}$.

(b) If $B=l_{p}$ (the set of all sequences $x=\left(x_{1}, x_{2}, \cdots\right)$, with $\sum_{i=1}^{\infty}\left|x_{i}\right|^{p}<\infty$ ) $p \geqq 2)$ then $\lambda \in\left(l_{p / 2}^{*}\right)^{+}$is adequate with $C_{\lambda}=\|\lambda\|_{p / p \text {. }}$.

If $\lambda \in l_{\times}^{+}$is adequate for $B$ then for that $\lambda$ we define the inner product

$$
(x, y)=\sum_{i=1}^{\infty} \lambda_{i} \beta_{i}(x) \beta_{i}(y) \text { for all } x, y \text { in } B .
$$


Let $\|x\|_{\lambda}=(x, x)^{\frac{1}{2}}$ then $\|x\|_{\lambda} \leqq C_{\lambda}\|x\|_{B}$. Let $H_{\lambda}$ be the Hilbert space obtained by the completion of $B$ under the norm $\|\cdot\|_{\lambda}$.

From (2.1) and the fact that $\beta_{n}$ is a $\|\cdot\|_{\lambda}$-continuous function on $B$, it follows that $\|x\|_{B}$ is a measurable function in $\|\cdot\|_{\lambda}$-topology, and hence $B$ is a $\|\cdot\|_{\lambda}$-measurable subset of $H_{\lambda}$. Therefore if $\mu$ is a measure on $B$, it can be regarded as a measure on $H_{\lambda}$ via $\mu(A)=\mu(A \cap B)$ for all $A \in \mathscr{B}\left(H_{\lambda}\right)$, Borel sets of $H_{\lambda}$.

The following ideas are given in Kuelbs and Mandrekar (1972).

If $\lambda \in l_{x}^{+}$and $\left\{\mu_{s}: s \in A\right\}$ is a family of probability measures on $B$ such that

$$
\mu_{s}\left\{x \in B: \sum_{n=1}^{\infty} \lambda_{n} \beta_{n}^{2}(x)<x\right\}=1
$$

for each $s \in A$, we say $\lambda$ is sufficient for the family $\left\{\mu_{s}: s \in A\right\}$.

Every $\lambda$ adequate for $B$ in $l_{x}^{+}$is sufficient for any family of probability measures on $B$.

A family of probability measures $\left\{\mu_{s}: s \in A\right\}$ on $B$ is a $\lambda$-family for some $\lambda \in l_{\times}^{+}$if $\lambda$ is sufficient for $\left\{\mu_{s}: s \in A\right\}$ and for every $\varepsilon, \delta>0$ there is a sequence $\left\{\varepsilon_{N}\right\}$ such that

$$
\mu_{s}\left\{x \in B: \sum_{n=N+1}^{\infty} \lambda_{n} \beta_{n}^{2}(x)<\delta\right\}>1-\varepsilon
$$

implies

$$
\mu_{\mathrm{s}}\left\{x \in B:\left\|\sum_{n=N+1}^{\infty} \beta_{n}(x) b_{n}\right\|_{B}<h(\delta)\right\}>1-\left(\varepsilon+\varepsilon_{N}\right) \text { for all } s,
$$

where $\lim _{N} \varepsilon_{N}=0$ and $h$ is a strictly increasing continuous function on $[0, \infty)$ with $h(0)=0$.

It is quite clear that any family of probability measures on a real separable Hilbert space is a $\lambda$-family with $\lambda=(1,1, \cdots)$ and $h(\delta)=\delta^{\frac{1}{2}}$.

Finally for $x \in B, N=1,2, \cdots$, we define

$$
P_{N} x=\sum_{k=1}^{N} \beta_{k}(x) b_{k}
$$

\section{Main result}

Let $\Gamma$ be a Gaussian measure with mean zero on $(B, \mathscr{B}(B))$. Then by an argument similar to Lemma 2.2 of (Kuelbs (1970)), $\Gamma$ is a Gaussian measure on $H_{\lambda}$, and therefore there exists a nonnegative, symmetric, trace class operator (often called an $S$-operator) $\rho$ on $H_{\lambda}^{*}$ into $H_{\lambda}$ such that 


$$
(\rho x, x)=\int_{H_{\lambda}}(y, x)^{2} d \Gamma(y)
$$

for $x \in H_{\lambda}^{*}$, and $\Gamma$ is uniquely determined on $H_{\lambda}$ by the operator $\rho$. We shall assume that $\rho$ has dense range. Let $t>0$ and let $\Gamma_{t}$ be a Gaussian measure on $H_{\lambda}^{*}$ with the covariance operator $t j \rho j^{*}$ where $j$ is the bijection map on $H_{\lambda}$ to $H_{\wedge}^{*}$ given by Riesz Representation Theorem. Let $C_{t}=\left(1+t j \rho j^{*}\right)^{\frac{1}{2}}$.

3.1. Theorem. A bounded continuous functional $\varphi$ on $B^{*}$ with $\varphi(0)=1$ is c.f. of a probability measure on $B$ if and only if

(i) the family of measures $\left\{\mu_{N}\right\}$ corresponding to $\varphi\left(P_{N}(\cdot)\right)$ is a $\lambda$-family for some $\lambda \in l_{x}^{+}$which is adequate for $B$,

(ii) $\left(\operatorname{det} C_{t}\right) \int_{B^{*}} \varphi(y) \exp \{i\langle x, y\rangle\} \Gamma_{t}(d y) \geqq 0$ for all $x \in B$, for all $t \geqq t_{0}$, some $t_{0}>0$,

(iii) $\lim _{y \rightarrow x} \lim _{t \rightarrow x}\left(\operatorname{det} C_{t}\right) \int_{B} \int_{B} \varphi(\gamma y) \exp \{i\langle x, y\rangle\} \Gamma_{1}(d y) \nu_{1}(d x)=\varphi(0)$, where $\nu_{1}$ is a Gauss measure on $B$.

Proof. Let $\mu$ be a probability measure on $B$ with c.f. $\varphi$. Then condition (i) holds for every $\lambda \in l_{x}^{+}$which is adequate for $B$ since $\left\{\mu_{N}\right\}$ is conditionally compact and Lemma 3.1 of Kuelbs and Mandrekar (1972) applies. Now let $\lambda \in l_{\infty}^{+}$be adequate for $B$ and consider the Hilbert space $H_{\lambda}$. Since $B$ is a measurable subset of $H_{\lambda}, \mu$ can be regarded as a probability measure on $H_{\lambda}$. Let $\psi$ be a c.f. of $\mu$ on $H_{\lambda}$. Then by Theorem 1 of Boylan (1975) we have

$$
\left(\operatorname{det} C_{\imath}\right) \int_{H_{i}^{*}} \psi(y) \exp \{i(x, y)\} \Gamma_{\imath}(d y) \geqq 0 \quad \text { for all } \quad x \in H_{\lambda},
$$

for all $t \geqq t_{0}$, some $t_{0}>0$, and

(3.3) $\lim _{\gamma \rightarrow 0} \lim _{t \rightarrow \infty}\left(\operatorname{det} C_{t}\right) \int_{H_{\lambda}} \int_{H_{\lambda}^{*}} \psi(\gamma y) \exp \{i(x, y)\} \Gamma_{t}(d y) \nu_{1}(d x)=\psi(0)$.

Let $\nu_{1}$ be a Gauss measure on $B$. Then in view of (3.2) and (3.3) and the fact that $H_{\lambda}^{*}$ is a measurable dense subset of $B^{*}$ it can be seen that (ii) and (iii) are satisfied.

Now assume (i), (ii), (iii) hold. Let $\psi$ be restriction of $\varphi$ over $H_{\lambda}^{*}$. Then, from (ii) and (iii) and the fact that $B$ is a measurable dense subset of $H_{\lambda}$, it follows that conditions (a) and (b) of Theorem 1 of Boylan (1975) are satisfied for $\psi$. Hence $\psi$ is c.f. of a probability measure $\mu$ on $H_{\lambda}$. Let $\mu_{N}=\mu \circ P_{N}^{-1}$, then $\left\{\mu_{N}\right\}$ is compact on $H_{\lambda}$ and $\mu_{N}$ converges weakly to $\mu$ on $H_{\lambda}$. This together with (i) imply that $\left\{\mu_{N}\right\}$ is compact on $B$ (Hamedani and Mandrekar (1973), Lemma 1.2.11). Hence $\mu_{N}$ converges weakly to $\mu$ on $B$ and $\mu(B)=1$. This implies that the c.f. of $\mu$ is $\varphi(x)$ and the theorem is proved. 


\section{Acknowledgement}

The author would like to thank Professor V. S. Mandrekar, for valuable discussion concerning this paper.

\section{References}

S. L. Boylan (1975), 'Positive definite functionals, function-space transforms and abstract Wiener spaces', Duke Math. J., 42, 549-557.

M. M. Day (1962), Normed linear spaces, Ind rev. ed. (Academic Press. New York: SpringerVerlag. Berlin). MR26\# 2847.

L. Gross (1963), 'Harmonic analysis on Hilbert space'. Mem. Amer. Math. Soc 46. MR28 \# 4304.

G. G. Hamedani and V. Mandrekar (1973), 'Inversion formulae for the probability measures on Banach spaces', Trans. Amer. Math. Soc., 180, 1+3-169.

J. Kuelbs (1970), 'Gaussian measures on a Banach space'. J. Functional Analysis 5, 354-367. MR41 \# 4639.

J. Kuelbs and V. Mandrekar (1972), 'Harmonic anlaysis on $F$-spaces with a basis'. Trans. Amer. Math. Soc. 169, 113-152.

V. Sazanov (1958), 'Remarks on characteristic functionals'. Teor. Verojatnost. i Primenen. 3, 201-205 = Theor. Probability Appl. 3 (1958), 188-192. MR20 \#4882.

Arya-Mehr University of Technology,

Tehran, Iran.

Michigan State University, U.S.A. 